\title{
Gingivectomy and flap operation procedure with papilla preservation technique in pyogenic granuloma patient
}

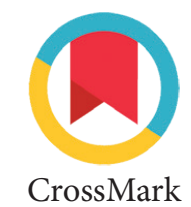

\author{
Sandra O. Kuswandani*
}

\section{Abstract}

Objective: Pyogenic granuloma is one of commonest gingival enlargement in oral cavity or skin, tumor-like, and non-neoplastic lesion. Eventhough, the exact etiology is unknown, but estimated result from chronic irritation or trauma.

Methods: This case reported a male patient, 28 years old with 15 milimetres in diameter gingival enlargement in middle anterior of upper jaw region, reddish, bleeding but without any pus. He is using toothpicks and suddenly impaled to the gingiva one week ago. The gingiva slowly enlarged in one week. Gingivectomy and flap operation procedure with papilla preservation technique were done with the intention of removing the lesion. Then, it sent to Pathology laboratorium to enforce the diagnosis.
Results: The histopathology result the lesion as pyogenic granuloma. After two weeks follow up, no reccurency for the lesion. The challenge to avoid gingival recession in anterior region is minimized with papilla preservation technique.

Conclusion: Pyogenic granuloma occur the most frequently in the anterior gingiva. The patient sometimes did not realize the main cause and the process of the enlargement until interfere the quality of life. Dentist should know the disease, the etiology and pathogenesis, and should understand when to refer to specialist.
Department of Periodontics, Faculty of Dentistry, University of Indonesia, Jakarta, Indonesia
*Corresponding to:

Sandra 0. Kuswandani, Department of Periodontics, Faculty of Dentistry, University of Indonesia, Jakarta, Indonesia sandra.0livia01@ui.ac.id

Received: 30 May 2018 Revised: 10 August 2018 Accepted: 7 November 2018 Available Online 1 December 2018

Keywords: Pyogenic granuloma, Gingival enlargement, Gingivectomy, Flap operation procedure, Papilla preservation Cite this Article: Kuswandani S0. 2018. Gingivectomy and flap operation procedure with papilla preservation technique in pyogenic granuloma patient. Journal of Dentomaxillofacial Science 3(3): 180-183. D0l: 10.15562/jdmfs.v3i3.740

\section{Introduction}

Pyogenic granuloma is one of commonest gingival enlargement in oral cavity or skin, tumor-like and non-neoplastic lesion. The appellation of "pyogenic granuloma". Eventhough, the exact etiology is unknown, but estimated result from chronic irritation or trauma, factor of hormonal or drug-induced. ${ }^{1,2}$

The prevalence of pyogenic granuloma in nonneoplastic lesion is $52.71 \%$ based on Shamim research in India. ${ }^{3}$ It is frequently occur in female then male, although it could see in all sex and age groups. Some literature mentioned pyogenic granuloma also as pregnancy granuloma, because predominantly seen in pregnant woman that related to hormonal changes.

The clinical appearance of pyogenic granuloma presented as the single lesion. The lesion is nodule or sessile papule, sometimes with smooth or lobulated surface. The mature lesion is haemorragic, as result as the decrease of vascularization. The size varied from millimeters to centimeters. ${ }^{1,2,4,5}$

Authors report a case of pyogenic granuloma managed by gingivectomy and flap operation procedure. The procedure combined with papilla preservation technique in order to maintain esthetic in anterior region and minimize the gingival recession after surgery.

\section{Methods Anamnesis}

This case reported a male patient, 28 years old come to Dental Hospital, Faculty of Dentistry, University of Indonesia with his chief complaint his anterior upper jaw gingiva enlarge within one week. His using tooth picks and suddenly impaled to the gingiva one week ago. The gingiva slowly enlarged in one week. At first, the gingiva seen normal, but gradually enlarge in size and sometimes bleeding when tooth-brushing. No complaint about pain, but discomfort when swallowing. The patient brushed his teeth twice daily using a toothbrush and paste, with horizontal movement. The general health condition was excellent, and no extra oral abnormalities.

\section{Clinical examination}

In visual examination, the size of the lesion around 15 milimetres in diameter, located in the middle anterior of upper jaw region, present in the interdental area between 11 and 21. The colour was reddish, smooth shiny in surface and also the margin is clear defined. In palpation examination, it was non tender, bleeding and haemorrhagic without any signs of exudation. Figure 1 Oral Hygiene index categorized as fair (1.5). 


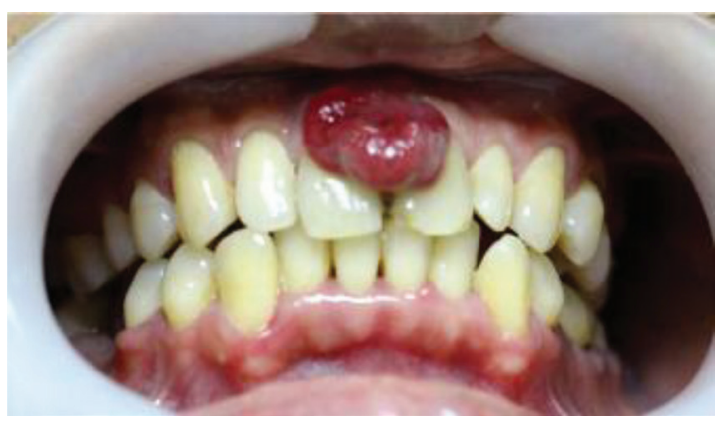

Figure 1 Enlargement gingiva between tooth 11 and 21

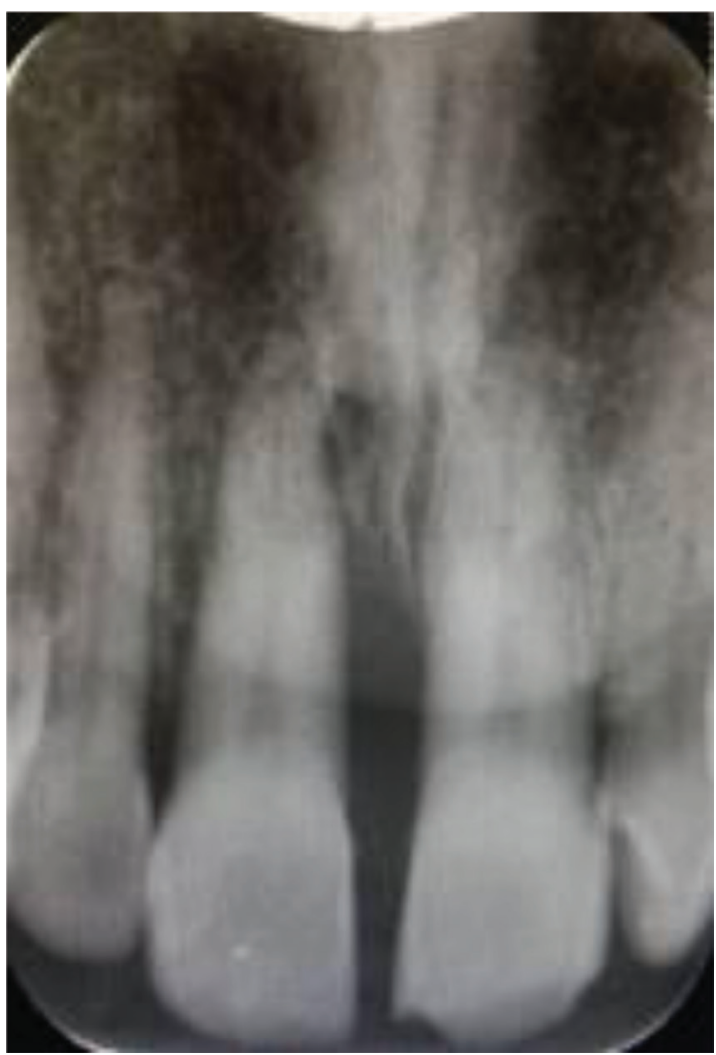

Figure 2 Radiographic examination. The bone destruction are seen in between 11 and 21
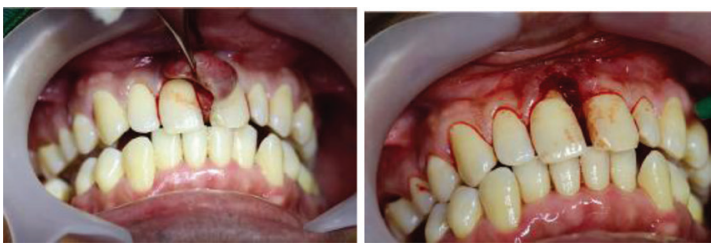

Figure 3 Before and after gingivectomy treatment

\section{Radiographic examination}

Radiographic examination showed the radioluency as horizontal bone loss between 11 and 21, and vertical bone loss in mesial 11. The thickening of lamina dura and periodontal space in tooth 11 and 21 . Figure 2.

\section{Results}

\section{Surgical Treatment}

Initial treatment performed by scaling and root planning. The goal is eliminate the plaque and calculus as the main factors. The patient was explained through dental health education about oral hygiene and guidance of using toothpicks. The patient also informed about the treatment planning of the possibility of surgery with gingivectomy and flap operation procedure. Concerning the anterior area, which required esthetic factor, the papilla preservation flap technique was planning to do.

After 10 days from the initial treatment, no significant changes for the lesion. Gingivectomy and flap operation procedure with papilla preservation technique was performed. Asepsis and local anesthesia was performed. Gingivectomy was performed to removing the lesion. Figure 3 the lesion was put in the formaline solution and sent to clinical pathology laboratorium to enforce the diagnosis. Figure 4.

The periodontal flap operation procedure was started with papilla preservation flap technique from mesial area tooth 13 to mesial area tooth 23. Figure 5 the flap was reflected, and the bone destruction already impaired to the buccal bone tooth 11 and 21, the destruction also continued to the surrounding distopalatal tooth 11. Figure 6 the granulation tissue, necrotic cementum, destruction bone was removed. Irigation by saline, $\mathrm{H}_{2} \mathrm{O}_{2} 3 \%$ and povidone iodine was performed. The flap reflected and suturing done by Nylon suture size 5.0. The patient instructed to maintain oral hygiene, and follow-up examination 1 week after surgery. The patient prescribed with $0.2 \%$ chlorexidine mouthwash and analgesics for 1 week. Figure 7.

After 2 week follow-up no reccurency of enlarge gingiva. The interdental gingiva still attached, with minimal gingival recession in distal tooth 11 , and mesial 21. The patient have not come to do follow up examination, but from the communication there is reccurency. Figure 8.

\section{Histopathological Examination}

The histophatological examination resulted as an ulcerative layer epithelium, with papilomatosis. Subepithelium described with vein blood vessels with endothelium cell. Acute inflammatory cell, lymphocyte, and plasma cell are seen in between connective tissue. The result is written as pyogenic granuloma, without any signs of malignancy. 


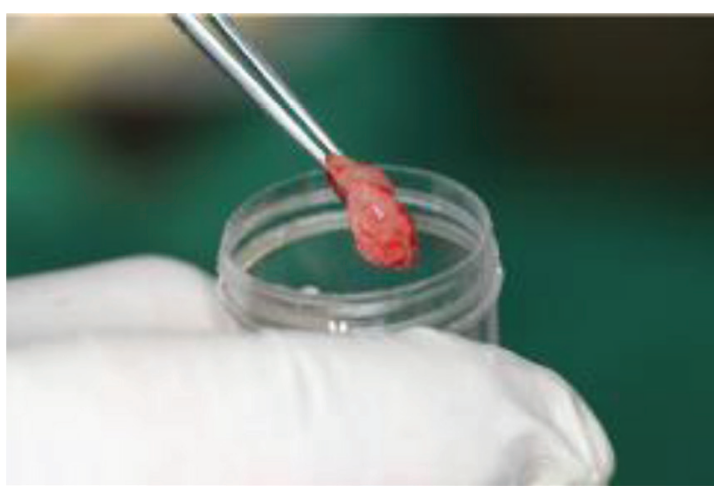

Figure 4 Excised lesions

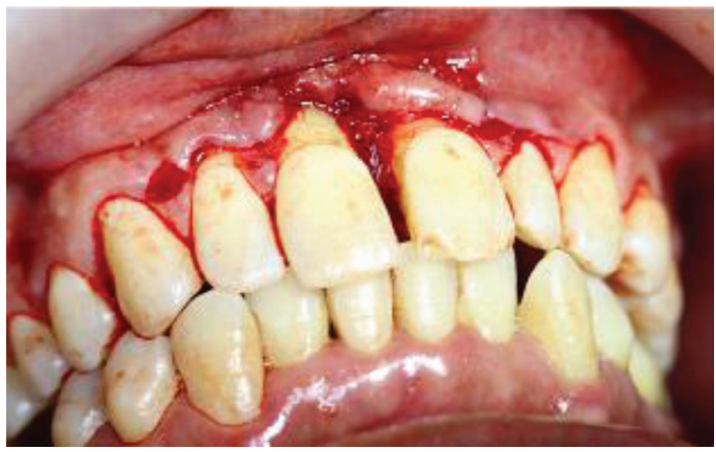

Figure 5 The papilla preservation incision flap

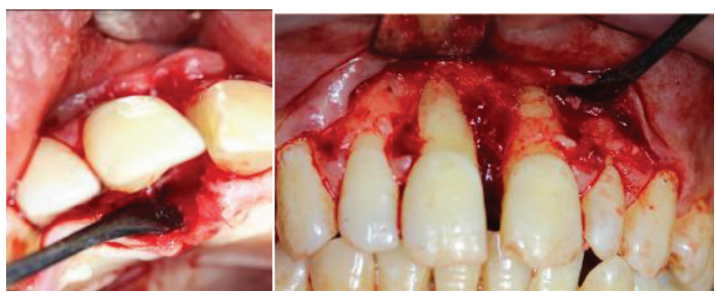

Figure 6 The bone destruction is seen as horizontal bone loss between tooth 11 and 21, and also in the surrounding bone tooth 11

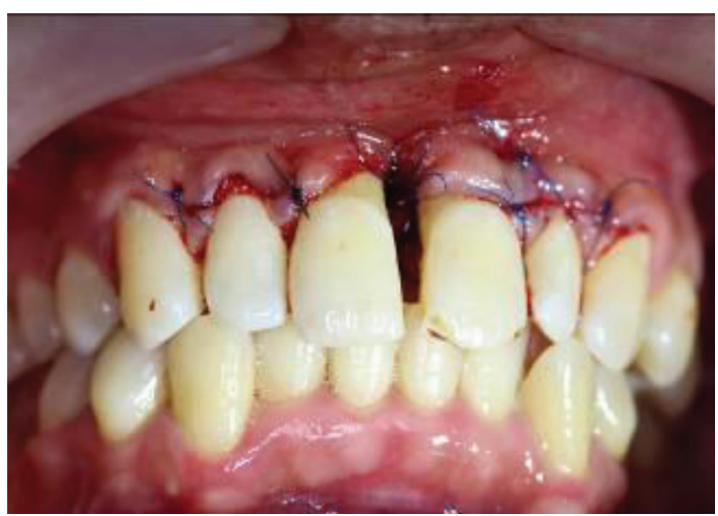

Figure 7 The flap reposition and suturing

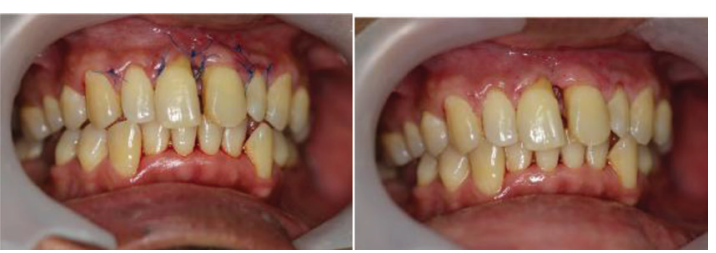

Figure 8 Two weeks follow-up. The suturing still intact, no recurrency in enlarge, slight hyperemia

\section{Discussion}

The prevalence of pyogenic granuloma in nonneoplastic lesion is $52.71 \%$ based on Shamim research in India. ${ }^{3}$ It is frequently occur in female then male, mainly termed as pregnancy granuloma related to hormonal changes. In this patient, pyogenic granuloma happen in male. The explanation from Bashkar and Jacoway study, resulted that pyogenic granuloma occurs almost as often in males as females. It is strengthen than maybe a hormonal basis is doubtful.,

Possibly, pyogenic granuloma originates as a response of tissues to minor trauma and/or chronic irritation, therefore it is role as invasion of nonspecific microorganisms, although microorganisms are seldom demonstrated within the lesion. In this patient the main etiology of gingival enlargement is misuse of toothpicks. The gingiva was injured shortly after. Study related between pyogenic granuloma and minor trauma mentioned that the bacteria role important causes. In kamal systematic review found that kerr, bhaskar, shafer conclude that staphylococci arises as a result of infection, these microorganism could produce colonies with fungus-like characteristics. Another reason that minor trauma provide a pathway for invasion for microorganism. The irritant may role as a stimulus, destructive agent or both. The type of inflammation formed in the pyogenic granuloma is destruction of fixed tissue cells is slight but stimulus to proliferation of vascular endothelium persists and exerts its influence over a long period of time. ${ }^{6}$

Surgical excision or gingivectomy is the treatment of choice. But, case report from Frumkin mentioned nonsurgical treatment of recurrent pyogenic granuloma as effective choices. The patient was given strict oral hygiene instructions, including the use of regular and single-tufted toothbrushes and an interdental brush until the achievement of optimal oral hygiene. The treatments. These treatments were done under local anesthesia, in combination with an adjunctive chlorhexidine rinse in three cycles of debridement in the following 
2 months. Following the treatment, the patient was followed up every 2 weeks for the first 2 months, and the enlarge gingiva reduced in 3 months. ${ }^{7}$ This report also gave us a caution to prevent reccurency the patient should strictly informed about maintain oral hygiene and dentist should have an ability to do dental health education to the patient. The non-surgical treatment also special consideration for treating a pregnant patient, although there is possibility to completed surgical treatment in second trimester. ${ }^{8}$

Flap operation procedure done because in radiographic examination already involving bone destruction. The periodontal breakdown in pyogenic granuloma conclude as mutual confounding and synergistic biological interactive effects with respect to the lesion size and microvessel density in the susceptible host that may be also associated with the bone resorption and tissue destruction. The Gonzales study after bivariate logistic regression analysis, only the evolution course $\geq 12$ months, moderate-to-severe inflammatory infiltrate density, and IL-4 SID score $\geq 8.04$ remained as robust predictors of periodontal damage. ${ }^{9}$ Papilla preservation techniques consider as anterior area in maxilla between tooth 11 and 21. A simple modification was tried by raising papilla preservation flap, including the growth in buccal flap and sectioning the pathologic tissue from underside of flap. This led to an esthetic, less invasive procedure with primary healing, reduced pain, also reduction possibility of the gingival recession in another tooth area including tooth 13,12, 22, 23 and also minimize the lesion area. ${ }^{10}$ Other treatment options include excision using lasers, cryosurgery, or electrodesiccation. These techniques would also result in large areas healing by secondary intention and limitations similar to excision. ${ }^{10}$

To differentiate pyogenic granuloma with the others lesion such as hemangioma, peripheral ossifying fibroma, Kaposi sarcoma, we should learn about the histopathology. The pyogenic granuloma divided into pyogenic granulomas are classified as the Lobular capillary haemangioma (LCH) type and the non- Lobular capillary haemangioma type. Sternberg et al. in Marla study informed three distinct phases to describe the course of pyogenic granuloma. The "early phase" reveals a compact cellular stroma with little lumen formation. The next phase described as the capillary phase reveals lobules which are highly vascular with abundant intraluminal red blood cells. The final phase referred to as "involutionary phase" shows intraand perilobular fibrosis. This phase is suggestive of healing phase of pyogenic granuloma. ${ }^{11}$ Differential diagnosis is done on clinical and histological features that help in adequate treatment and prognosis. A recurrence rate of $16 \%$ however has been reported. ${ }^{11}$

\section{Conclusion}

Pyogenic granuloma occur the most frequently in the anterior gingiva. The patient sometimes did not realize the main cause and the process of the enlargement until interfere the quality of life. Dentist should know the disease, the etiology and pathogenesis, and should understand when to refer to specialist.

\section{Acknowledgment}

The authors stated no funding to declare.

\section{Conflict of Interest}

The authors report no conflict of interest.

\section{References}

1. Shivanaikar S, Patil S. Pyogenic granuloma. Guident 2015;8: 65-67.

2. Mastammanavar D, Hunasgi S, Koneru A, et al. Aggressive pyogenic granuloma: a case report. Int J Oral Maxillofac Pathol 2014;5: 29-32.

3. Shamim T, Varghese VI, Shameena PM, et al. A retrospective analysis of gingival biopsied lesions in south indian population: 2001-2006. Med Oral Patol Oral Cir Bucal 2008;13: E414-E418.

4. Gomes SR, Shakir QJ, Thaker PV, et al. Pyogenic granuloma of the gingiva: a misnomer? a case report and review of literature. J Indian Soc Periodontol 2013;17: 514-519.

5. Tjiptoningsih UG. Enlargement gingival treatment on teeth 11 and 21. J Dentomaxillofac Sci 2016;1: 196-200.

6. Kamal R, Dahiya P, Puri A. Oral pyogenic granuloma: various concepts of etiopathogenesis. J Oral Maxillofac Pathol 2012;16: 79-82.

7. Frumkin N, Nashef R, Shapira L, et al. Nonsurgical treatment of recurrent gingival pyogenic granuloma : a case report. Quintessence Int 2015;46: 539-544.

8. Assaf M. Oral pyogenic granuloma: a literature review and case report. SDJ 2014;9: 18-21.

9. González-Pérez L, Isaza-Guzmán D, Tobón-Arroyav S. Association study between clinicopathological variables and periodontal breakdown in gingival pyogenic granuloma. Clin Oral Investig 2014;18: 2137-2149.

10. Tiwari S, Neelakanti A, Sathyanarayana S. Pyogenic granuloma and modified laser excision. J Indian Soc Periodontol 2017;21: 241-244.

11. Marla V, Shrestha A, Goel K, et al. The histophatological spectrum of pyogenic granuloma: a case series. Case Rep Dent 2016;2016: 1-6.

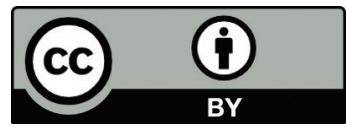

This work is licensed under a Creative Commons Attribution 